\title{
Scale Effects of Physical Modelling of Anaerobic Treatment Plants in the Treatment of Cassava Wastewater
}

\section{E. Onukwugha1, B.C.Okoro² , J.C. Agunwamba3 ${ }^{3}$ A.O. Ibeje ${ }^{4}$}

\author{
${ }^{1}$ Department of Civil Engineering, Federal Polytechnic, Nekede, Owerri, Nigeria \\ ${ }^{2}$ Department of Civil Engineering, Federal University of Technology, Owerri, Nigeria \\ ${ }^{3}$ Department of Civil Engineering, University of Nigeria, Nsukka \\ ${ }^{4}$ Department of Civil Engineering, Imo State University, Owerri, Nigeria
}

\begin{abstract}
Three Anaerobic Baffled Reactors (ABRs) are used to evaluate the extent of scale effects of cyanide inhibition of cassava wastewater treatment. The reactors (physical models) have aspect ratios of (53:16:30; 4:1:1; 10:3:6). Kinetic analyses of specific growth rate $\boldsymbol{\mu}_{\boldsymbol{m a x}}$ and half saturation constant $\boldsymbol{k}_{\boldsymbol{s}}$ are evaluated for the respective reactors. For the respective reactors, non-inhibited cassava wastewater treatment process showed Kincannon model yields $\left(\mu_{\max }=17.24\right.$ day $^{-1} ; 21.74$ day $^{-1} ; 21.28$ day $^{-1}$

and $\left.k_{s}=1.00 \mathrm{mgCODL}^{-1} ; 2.37 \mathrm{mgCODL}^{-1} ; 1.23 \mathrm{mgCODL}^{-1}\right)$ while Monod model yields $\left(\mu_{\max }=10.87 \mathrm{day}^{-1}\right.$; 12.82 day $^{-1} ; 13.70$ day $^{-1}$ and $\left.k_{s}=0.87 \mathrm{mgCODL}^{-1} ; 1.92 \mathrm{mgCODL}^{-1} ; 2.32 \mathrm{mgCODL}^{-1}\right)$. Coefficient of determination $R^{2}$ is used to verify the respective models to yield values of $\left(\boldsymbol{R}^{2}=\mathbf{0 . 8 3 9} ; \mathbf{0 . 9 6 0 ; 0 . 9 4 8}\right)$ for Kincannon model and $\left(\boldsymbol{R}^{2}=\mathbf{0 . 9 1 7} ; \mathbf{0 . 8 4 2} ; \mathbf{0 . 8 3 0}\right)$ for Monod model.
\end{abstract}

Keywords: Anaerobic, Treatment, Cassava Wastewater, Monod model and Kincannon model

\section{Introduction}

Cassava (Manihot esculenta crantz, also known as manioc or yucca) is one of the leading food and feed plants in the world: it ranks fourth among staple crops with a global production of about 160 milllion tons per year (1). Most of these are grown in three regions, West Africa, and the Congo basin, tropical South America, and South East Asia (2), while in Western countries it is not commonly used, because of the presence of cyanoglucosides (linamarin and lotaustralin).

Nigeria currently is the largest producer of cassava in the whole world with an annual output of over 34 million tons of tuberous root and it plays a dominant role in the rural economy in the southern agroecological zone (3). As a food crop, cassava fits well into the farming system of the small holder farmers in Nigeria because it is available year round, thus providing household food security. Cassava tubers can be kept in the ground prior to harvesting up to two years but once harvested, they begin to deteriorate. To forestall early deterioration and also due to its bulky nature, cassava is usually traded in their processed form. The bulky roots contain much moisture (60-65\%), making their transportation from rural areas difficult and expensive. Processing the tuber into a dry form reduces the moisture content and converts into a more durable and stable product with less volume which makes it more transportable $(4 ; 5)$. Over the years cassava has been transformed into a number of product both for domestic use (depending on local customs and preference) and industrial uses. Cassava in the fresh form contains cyanide which is extremely toxic to humans and animals and therefore needs to be processed to reduce the cyanide content to safe level (6). Traditional cassava processing method involve several steps including peeling, soaking, grinding, steeping in water and left in air to allow fermentation to occur, drying, milling, roasting, steaming, pounding and mixing in cold or hot water. The wastewater from cassava processing or its derivative (garri) ends up with domestic sewage if processed in small quantities while others end up being carried with industrial wastes if processed in large industrial quantities. Lastly, others percolate into the soil depending on the processors. Wastewater from cassava processing, if released directly into the environment before proper treatment, is a source of pollution. This effect on the

This article is published under the terms of the Creative Commons Attribution License 4.0

Author(s) retain the copyright of this article. Publication rights with Alkhaer Publications.

Published at: http://www.ijsciences.com/pub/issue/2016-11/

DOI: 10.18483/ijSci.1127; Online ISSN: 2305-3925; Print ISSN: 2410-4477 
environment is yet to be addressed properly in developing countries due to inadequate equipment and lack of research materials.

A great number of recent studies have reported many biotechnological approaches to improve the safety and quality of cassava flour $(7 ; 8 ; 9)$, and the effect of different processing modalities of the tuberous roots on the level of these toxic substances and functional properties has been assessed $(10 ; 11 ; 12)$. The objective of this study is to study the scale effect of a physical model of anaerobic treatment plants in the treatment of cassava wastewater as well as to develop a mathematical model describing the microbial kinetics.

\section{Materials and Method}

\subsection{The Reactor}

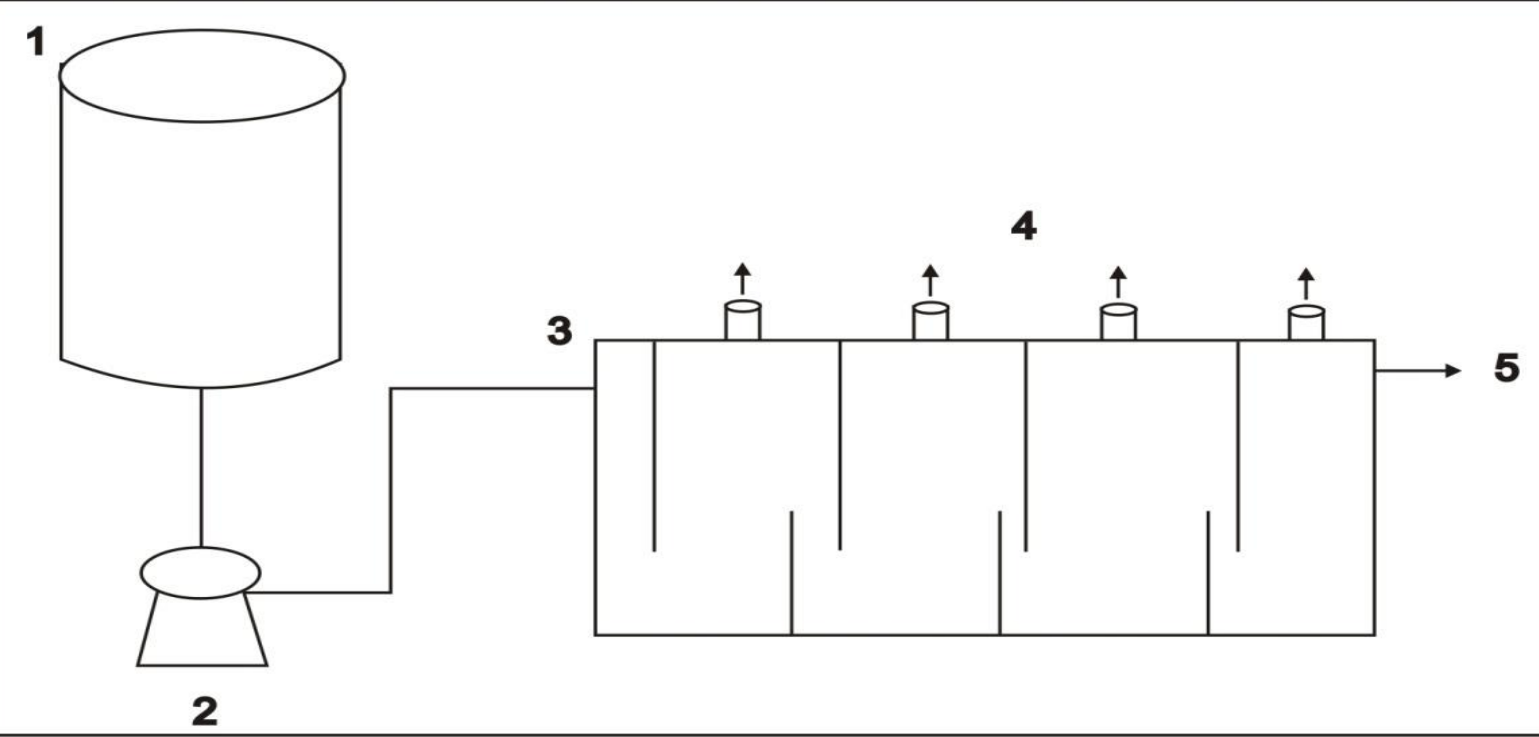

Fig. 1: Scheme of the ABR. 1. Feed Tank; 2. Peristaltic Pump; 3. Influent; 4. Sampling Ports; 5. Effluent.

The laboratory scale ABR was constructed from $6 \mathrm{~mm}$ thick stainless steel, with external dimensions of lengths, widths, depths and working volumes of three different sizes as shown in table 1. Fig. 1 shows a schematic diagram of the reactor. The reactors were divided into different number of equal compartments by vertical baffles with each compartment of each the reactors having downcomer and riser regions created by a further vertical baffle. For each of the reactors, the widths of upcomers were multiples of the widths of downcomer. The lower parts of the downcomer baffles were angled at $45^{\circ}$ in order to direct the flow evenly through the upcomer. This produced effective mixing and contact between the wastewater and anaerobic sludge at the base of each riser. Each compartment was equipped with sampling ports that allowed biological solids and liquid samples to be withdrawn. The operating temperature was maintained constant at $35 \pm 0.5^{\circ} \mathrm{C}$ by putting the reactor in a water bath equipped with a temperature regulator. The influent feed was pumped using variable speed peristaltic pump. The outlet was connected to a glass U-tube for level control and to trap solids.

Table 1: Dimensions of the Different Reactor Model Sizes.

\begin{tabular}{|l|c|c|c|}
\hline Dimensions & Reactor 1 & Reactor 2 & Reactor 3 \\
\hline Length (cm) & $\mathbf{5 3}$ & $\mathbf{4 0}$ & $\mathbf{5 0}$ \\
\hline Width (cm) & $\mathbf{1 6}$ & $\mathbf{1 0}$ & $\mathbf{1 5}$ \\
\hline Depth (cm) & $\mathbf{3 0}$ & $\mathbf{1 0}$ & $\mathbf{3 0}$ \\
\hline Working Volume (L) & $\mathbf{1 3 . 5 7}$ & $\mathbf{4}$ & $\mathbf{1 0}$ \\
\hline Upcomer Width/ Downcomer Width & $\mathbf{2 . 6}$ & $\mathbf{4}$ & $\mathbf{2 . 4}$ \\
\hline No of Compartments & $\mathbf{5}$ & $\mathbf{4}$ & $\mathbf{5}$ \\
\hline Volume of Each Compartment (L) & $\mathbf{2 . 7}$ & $\mathbf{2}$ & $\mathbf{2}$ \\
\hline
\end{tabular}

\subsection{Start-up of ABR}

Start-up without seed sludge was rather difficult and time consuming for suspended growth anaerobic reactors. The following 3 steps were taken: (i) the reactor was filled with cassava wastewater and allowed to rest for 15 days (ii) the sludge bed was allowed through a process of sludge accumulation by settling and sludge improvement and (iii) after 15 
days, feeding of the wastewater was resumed at a flow rate of 5.33litres per day and HRT of 6 days with a very low organic loading rate (OLR) of $0.067 \mathrm{kgCOD} / \mathrm{m}^{3}$.day. The resumed wastewater feeding is helped the development of sludge bed at the bottom of individual chambers of the ABR. This process of feeding the system followed by two weeks rest is based on the experiment made in Kanpur (India) for the start-up of a UASB plant without inoculum (13).

\subsection{Characterization of Wastewater}

The cassava wastewater from a cassava processing factory at Imo Polytechnic Umuagwo in eastern Nigeria was used as feed. The supernatant of the wastewater after the simple gravity settling, used in the investigation, had low TSS, as approximately $90 \%$ of the solids were removed. The supernatant wastewater was diluted to achieve the COD concentration required for each loading rate with water. In order to achieve $\mathrm{pH}$ and alkalinity adjustment, the supernatant was neutralized by $\mathrm{NaOH}$ and $\mathrm{NaHCO}_{3}$. A COD: N: $\mathrm{P}$ ratio of 300:5: 1 was kept during operation using $\mathrm{NH}_{4} \mathrm{Cl}$ and $\mathrm{K}_{2} \mathrm{HPO}_{4}$. The micro-nutrient deficiency was added occasionally to correct growth conditions according to (14).

\subsection{Procedure for Experiment}

The wastewater was collected twice a day from the cassava processing plant, and it was intermittently mixed to feed the reactor with a consistent quality. The wastewater came from processing cassava specie (bogot) that had no cyanide content (Table 2). The wastewater was fed to the reactor with the help of a variable speed peristaltic pump. The ABR was operated at various hydraulic retention times (HRTs) by varying the flow rate of influent wastewater $\left(Q_{\text {inf }}\right)$, thereby varying the organic loading rate (OLR). The wastewater flowed from the downcomer to the upcomer within an individual chamber through the sludge bed formed at the bottom of the individual chambers. After receiving treatment in the particular chamber, wastewater entered the next chamber from the top. Due to the specific design and .positioning of the baffle, the wastewater is evenly distributed in the upcomer and the vertical upflow velocity $\left(\mathrm{V}_{\text {up }}\right)$ could be significantly reduced. The treated effluent was collected from the outlet of the 3rd compartment (C3). The reactor was kept in a temperature controlled chamber maintained at $35{ }^{\circ} \mathrm{C}$.

\subsection{Mathematical Model Formulation \\ 2.5.1 Nomenclature}

$\mathrm{S}_{\mathrm{i}}=$ Substrate concentration in the influent $\left(\mathrm{mgl}^{-1}\right)$; $\mathrm{S}_{\mathrm{e}}=$ Substrate concentration in the effluent $\left(\mathrm{mgl}^{-1}\right)$; $\mathrm{k}_{\mathrm{s}}=$ Half saturation constant $\left(\mathrm{mgl}^{-1}\right) ; \mu=$ Specific growth rate of organism (per day); $\mu_{\max }=$ Maximum specific growth rate of organism (per day); $\mathrm{X}=$ concentration of active biomass $(\mathrm{mg} / \mathrm{L}) ; \mathrm{r}_{\mathrm{A}}=$ Rate of utilization of substrate (mg/l.day)

\subsubsection{Monod Model for ABR}

The Monod model is described as:

$$
\begin{aligned}
& r_{A}=\frac{d s}{d t}=\frac{Q}{V}\left(S_{i}-S_{e}\right)=\mu . X \\
= & \frac{\mu_{\max } S_{e}}{K_{s}+S_{e}} X=\frac{X V}{Q\left(S_{i}-S_{e}\right)}=
\end{aligned}
$$

Applying experimental results to Equations (2), graph will be plotted. In this, graph $\mathrm{XV} /\left(\mathrm{S}_{\mathrm{i}}-\mathrm{S}_{\mathrm{e}}\right)$ is plotted against $1 / \mathrm{S}_{\mathrm{e}}$

\begin{tabular}{|c|c|c|c|c|c|}
\hline Variety & $\begin{array}{l}\text { Total Leaves } \\
\text { Cyanide }(\mu \mathrm{g} / \mathrm{g})\end{array}$ & $\begin{array}{l}\text { Free Leaves } \\
\text { Cyanide }(\mu \mathrm{g} / \mathrm{g})\end{array}$ & $\begin{array}{l}\text { Total Roots } \\
\text { Cyanide }(\mu \mathrm{g} / \mathrm{g})\end{array}$ & $\begin{array}{ll}\text { Free } & \text { Roots } \\
\text { Cyanide }(\mu \mathrm{g} / \mathrm{g})\end{array}$ & $\begin{array}{l}\text { Total Cyanide } \\
\text { Content Ratio in } \\
\text { Leaves and Roots }\end{array}$ \\
\hline Java Brown & 490 & $33(6.7)$ & 185 & $9(4.9)$ & 2.6 \\
\hline Datu & 541 & $19(3.5)$ & 120 & $9(7.5)$ & 4.5 \\
\hline Bogot & 456 & $21(4.6)$ & n.d. & n.d. & n.d. \\
\hline Lakan & 189 & $13(6.9)$ & 45 & $0.4(1.0)$ & 4.2 \\
\hline
\end{tabular}

Table 2: Cassava Species of varying Cyanide Concentrations

n.d. = not detected; Source: 15 . 
3. Results and Discussion

3.1 Mathematical Model Calibration

Table 3: Computations for Reactor 1

\begin{tabular}{|c|c|c|c|c|c|c|}
\hline $\mathbf{S} / \mathbf{N}$ & $\begin{array}{l}x_{i} \\
=S V_{i} \\
-S V_{e} \\
(\mathbf{m g} / \\
\text { L.d) }\end{array}$ & $\frac{Q\left(S_{i}-S_{e}\right)}{\begin{array}{l}V \\
(\mathbf{m g} /\end{array}}$ & $\begin{array}{c}\frac{V}{Q\left(S_{i}-S_{e}\right)} \\
(\mathbf{m g} / \\
\text { L.d) }\end{array}$ & $\begin{array}{c}y= \\
x . \bar{V} \\
Q\left(S_{i}-S_{e}\right) \\
(\mathbf{m g} / \\
\text { L.d) }\end{array}$ & $\begin{array}{c}\begin{array}{c}S_{e} \\
(\mathrm{mg} / \mathrm{L}\end{array} \\
\end{array}$ & $\begin{array}{l}x=\frac{1}{S_{e}} \\
(\mathrm{~L} / \mathrm{mg})\end{array}$ \\
\hline 1 & 0.9 & 7.6 & 0.1315789 & 0.1184211 & 1.3 & 0.7692308 \\
\hline 2 & 0.9 & 7.8 & 0.1282051 & 0.1153846 & 1.5 & 0.6666667 \\
\hline 3 & 1.5 & 9 & 0.1111111 & 0.1666667 & 1.7 & $\mathbf{0 . 5 8 8 2 3 5 3}$ \\
\hline 4 & 1.5 & 9.5 & 0.1052632 & 0.1578947 & 1.8 & 0.5555556 \\
\hline 5 & 1.6 & 10.2 & 0.0980392 & 0.1568627 & 2 & 0.5 \\
\hline 6 & 1.6 & 12.8 & 0.078125 & 0.125 & 2.2 & 0.4545455 \\
\hline 7 & 1.7 & 13 & 0.0769231 & 0.1307692 & 2.5 & 0.4 \\
\hline 8 & 1.8 & 13.5 & 0.0740741 & $\mathbf{0 . 1 3 3 3 3 3 3}$ & 2.8 & 0.3571429 \\
\hline 9 & 1.8 & 14 & 0.0714286 & 0.1285714 & 3 & 0.3333333 \\
\hline 10 & 1.9 & 15 & 0.0666667 & 0.1266667 & 3.2 & 0.3125 \\
\hline 11 & 1.9 & 16 & 0.0625 & 0.11875 & 3.4 & 0.2941176 \\
\hline 12 & 2 & 18.4 & 0.0543478 & 0.1086957 & 3.6 & 0.2777778 \\
\hline 13 & 2 & 18.6 & 0.0537634 & 0.1075269 & 3.8 & 0.2631579 \\
\hline 14 & 2 & 18.8 & 0.0531915 & $\mathbf{0 . 1 0 6 3 8 3}$ & 4.8 & 0.2083333 \\
\hline 15 & 2.1 & 20 & 0.05 & 0.105 & 5.6 & 0.1785714 \\
\hline 16 & 2.1 & 20.4 & 0.0490196 & 0.1029412 & 5.8 & 0.1724138 \\
\hline 17 & 2.1 & 20.6 & 0.0485437 & 0.1019417 & 6.4 & 0.15625 \\
\hline 18 & 2.2 & 20.8 & 0.0480769 & 0.1057692 & 6.8 & 0.1470588 \\
\hline 19 & 2.2 & 20.8 & 0.0480769 & 0.1057692 & 7.5 & $\mathbf{0 . 1 3 3 3 3 3 3}$ \\
\hline 20 & 2.3 & 22.6 & 0.0442478 & 0.1017699 & 8.4 & 0.1190476 \\
\hline 21 & 2.3 & 24.2 & 0.0413223 & 0.0950413 & 8.8 & 0.1136364 \\
\hline 22 & 2.3 & 24.8 & 0.0403226 & 0.0927419 & 9.2 & 0.1086957 \\
\hline 23 & 2.4 & 26 & 0.0384615 & 0.0923077 & 10.8 & 0.0925926 \\
\hline 24 & 2.4 & 26.2 & 0.0381679 & 0.0916031 & 12.6 & 0.0793651 \\
\hline
\end{tabular}




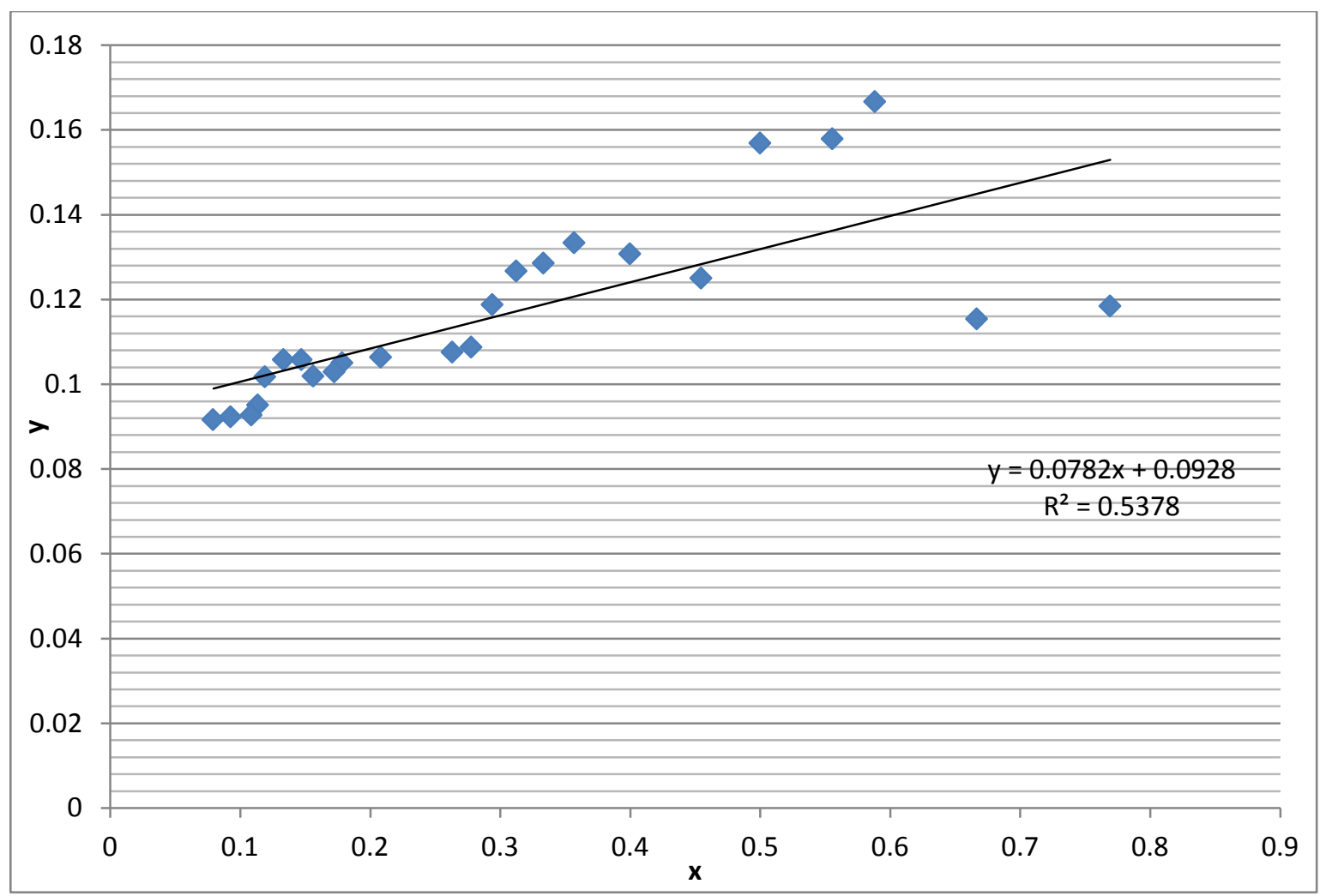

Fig. 2: A Plot of $y=\frac{x V}{Q\left(s_{i}-S_{e}\right)}(\mathrm{L.d} / \mathrm{mg})$ Versus $x=\frac{1}{s_{e}}(\mathrm{~L} / \mathrm{mg})$ for Reactor 1

From linear regression;

By comparison with equation 2;

$$
y=m x+c \equiv \boldsymbol{y}=\mathbf{0 . 0 7 8 x}+\mathbf{0 . 0 9 2}
$$

$$
\begin{aligned}
& \frac{1}{\mu_{\max }}=c=\text { intercept } ; \text { i.e. } \mu_{\max }=\frac{1}{c}=\frac{1}{0.092}=\mathbf{1 0 . 8 7} / \text { day } \\
& \text { and; } \quad \frac{K_{s}}{\mu_{\max }}=m=\text { slope; i.e. } K_{s}=\mu_{\max } * m \\
& \therefore K_{s}=10.87 * 0.078=\mathbf{0 . 8 7 m g C O D} / \mathbf{L}
\end{aligned}
$$

Substituting $\mu_{\max }$ and $\mathrm{k}_{\mathrm{s}}$ into equation 1 gives;

$$
r_{A}=\frac{d s}{d t}=\frac{Q}{V}\left(S_{i}-S_{e}\right)=\frac{\mu_{\max } * S_{e}}{K_{s}+S_{e}}=\frac{10.87 * \boldsymbol{S}_{e}}{\mathbf{0 . 8 7}+\boldsymbol{S}_{\boldsymbol{e}}}
$$


Table 4: Computations for Reactor 2

\begin{tabular}{|c|c|c|c|c|c|c|}
\hline $\mathbf{S} / \mathbf{N}$ & $\begin{array}{l}x_{i} \\
=S V_{i} \\
-S V_{e} \\
(\mathrm{mg} / \\
\text { L.d) } \\
\end{array}$ & $\frac{Q\left(S_{i}-S_{e}\right)}{V}\left(\begin{array}{c}V \\
\text { L.d })\end{array}\right.$ & $\begin{array}{c}\frac{V}{Q\left(S_{i}-S_{e}\right)} \\
(\mathbf{m g} / \\
\text { L.d) }\end{array}$ & $\begin{array}{c}y= \\
x . V \\
Q\left(S_{i}-S_{e}\right) \\
(\mathbf{m g} / \\
\text { L.d) }\end{array}$ & $\begin{array}{c}S_{e} \\
(\mathrm{mg} / \mathrm{L}\end{array}$ & $\begin{array}{l}x=\frac{1}{S_{e}} \\
(\mathrm{~L} / \mathrm{mg})\end{array}$ \\
\hline 1 & 1 & 6.4 & 0.15625 & 0.15625 & 1.5 & 0.6666667 \\
\hline 2 & 1 & 6.5 & 0.1538462 & 0.1538462 & 1.5 & 0.6666667 \\
\hline 3 & 1.5 & 6.8 & 0.1470588 & 0.2205882 & 1.6 & 0.625 \\
\hline 4 & 1.5 & 7 & 0.1428571 & 0.2142857 & 1.6 & 0.625 \\
\hline 5 & 1.6 & 9.8 & 0.1020408 & 0.1632653 & 1.8 & 0.5555556 \\
\hline 6 & 1.6 & 12 & $\mathbf{0 . 0 8 3 3 3 3 3}$ & $\mathbf{0 . 1 3 3 3 3 3 3}$ & 2.2 & 0.4545455 \\
\hline 7 & 1.8 & 14.6 & 0.0684932 & 0.1232877 & 2.5 & 0.4 \\
\hline 8 & 1.8 & 14.8 & 0.0675676 & 0.1216216 & 2.6 & 0.3846154 \\
\hline 9 & 1.8 & 15 & 0.0666667 & 0.12 & 2.8 & 0.3571429 \\
\hline 10 & 2 & 15.6 & 0.0641026 & 0.1282051 & 3 & $\mathbf{0 . 3 3 3 3 3 3 3}$ \\
\hline 11 & 2 & 17 & 0.0588235 & 0.1176471 & 3.2 & 0.3125 \\
\hline 12 & 2.2 & 19 & 0.0526316 & 0.1157895 & 3.3 & 0.3030303 \\
\hline 13 & 2.2 & 20.5 & 0.0487805 & 0.1073171 & 3.4 & 0.2941176 \\
\hline 14 & 2.3 & 18.8 & 0.0531915 & 0.1223404 & 3.6 & 0.2777778 \\
\hline 15 & 2.3 & 20 & 0.05 & 0.115 & 4.2 & 0.2380952 \\
\hline 16 & 2.4 & 20.4 & 0.0490196 & 0.1176471 & 4.8 & 0.2083333 \\
\hline 17 & 2.4 & 20.6 & 0.0485437 & 0.1165049 & 5.8 & 0.1724138 \\
\hline 18 & 2.4 & 20.8 & 0.0480769 & 0.1153846 & 6.6 & 0.1515152 \\
\hline 19 & 2.4 & 20.8 & 0.0480769 & 0.1153846 & 7.8 & 0.1282051 \\
\hline 20 & 2.3 & 22.6 & 0.0442478 & 0.1017699 & 8 & 0.125 \\
\hline 21 & 2.3 & 25 & 0.04 & 0.092 & 8.4 & 0.1190476 \\
\hline 22 & 2.3 & 26.4 & 0.0378788 & 0.0871212 & 10.6 & 0.0943396 \\
\hline 23 & 2.4 & 27.5 & 0.0363636 & 0.0872727 & 11.6 & 0.0862069 \\
\hline 24 & 2.6 & 28 & 0.0357143 & 0.0928571 & 16.6 & 0.060241 \\
\hline
\end{tabular}




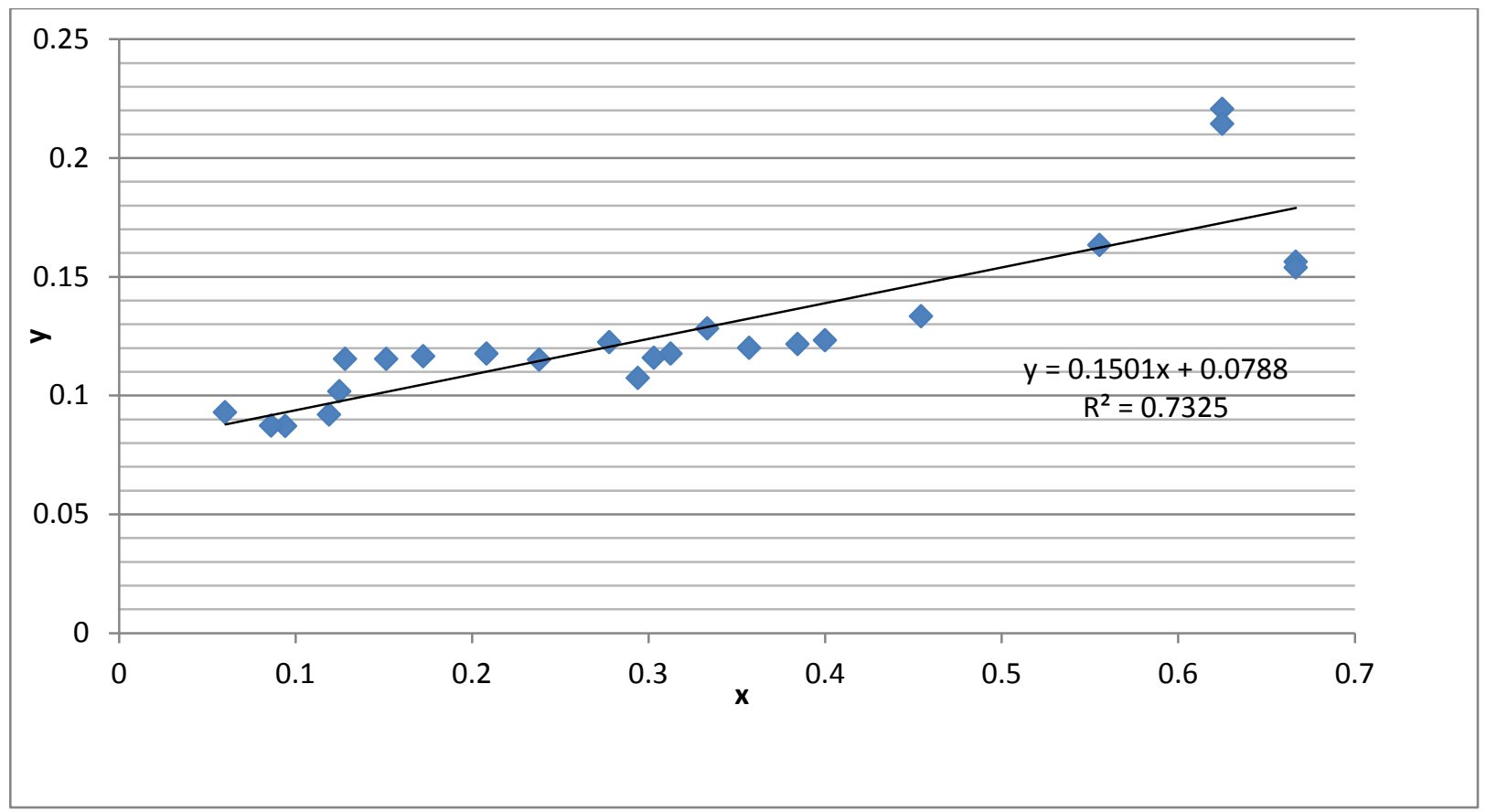

Fig. 3: A Plot of $y=\frac{X V}{Q\left(S_{i}-S_{e}\right)}(/ \mathrm{L} . \mathrm{d} / \mathrm{mg})$ Versus $x=\frac{1}{s_{e}}(\mathrm{~L} / \mathrm{mg})$ for Reactor 2

From linear regression equation;

By comparison with equation 2;

$$
y=m x+c \equiv \boldsymbol{y}=\mathbf{0 . 1 5 0} \boldsymbol{x}+\mathbf{0 . 0 7 8}
$$

$$
\begin{aligned}
& \frac{1}{\mu_{\max }}=c=\text { intercept; i.e. } \mu_{\max }=\frac{1}{c}=\frac{1}{0.078}=\mathbf{1 2 . 8 2} / \text { day } \\
& \text { and; } \quad \frac{K_{s}}{\mu_{\max }}=m=\text { slope; i.e. } K_{s}=\mu_{\max } * m \\
& \therefore K_{S}=12.82 * 0.150=1.92 \mathrm{mgCOD} / \mathrm{L}
\end{aligned}
$$

Substituting $\mu_{\max }$ and $\mathrm{k}_{\mathrm{s}}$ into equation 1 gives;

$$
r_{A}=\frac{d s}{d t}=\frac{Q}{V}\left(S_{i}-S_{e}\right)=\frac{\mu_{\max } * S_{e}}{K_{s}+S_{e}}=\frac{12.82 * \boldsymbol{S}_{e}}{1.92+\boldsymbol{S}_{e}}
$$


Table 5: Computations for Reactor 3

\begin{tabular}{|c|c|c|c|c|c|c|}
\hline $\mathbf{S} / \mathbf{N}$ & $\begin{array}{l}x_{i} \\
=S V_{i} \\
-S V_{e} \\
(\mathbf{m g} / \\
\text { L.d) }\end{array}$ & $\frac{Q\left(S_{i}-S_{e}\right)}{V}\left(\begin{array}{c}V \\
(\mathrm{mg} / \\
\text { L.d })\end{array}\right.$ & $\begin{array}{c}\frac{V}{Q\left(S_{i}-S_{e}\right)} \\
(\mathbf{m g} / \\
\text { L.d) }\end{array}$ & $\begin{array}{c}y= \\
x_{i} \cdot V \\
Q\left(S_{i}-S_{e}\right) \\
(\mathbf{m g} / \\
\text { L.d) }\end{array}$ & $\begin{array}{c}S_{e} \\
(\mathrm{mg} / \mathrm{L}\end{array}$ & $\begin{array}{l}x=\frac{1}{S_{e}} \\
(\mathrm{~L} / \mathrm{mg})\end{array}$ \\
\hline 1 & 1.2 & 5.8 & 0.1724138 & 0.2068966 & 1.2 & $\mathbf{0 . 8 3 3 3 3 3 3}$ \\
\hline 2 & 1.3 & 6 & 0.1666667 & 0.2166667 & 1.4 & 0.7142857 \\
\hline 3 & 1.3 & 6 & 0.1666667 & 0.2166667 & 1.4 & 0.7142857 \\
\hline 4 & 1.3 & 6.8 & 0.1470588 & 0.1911765 & 1.4 & 0.7142857 \\
\hline 5 & 1.4 & 7.4 & 0.1351351 & 0.1891892 & 1.8 & 0.5555556 \\
\hline 6 & 1.4 & 8.6 & 0.1162791 & 0.1627907 & 2 & 0.5 \\
\hline 7 & 1.5 & 14 & 0.0714286 & 0.1071429 & 2 & 0.5 \\
\hline 8 & 1.5 & 14.2 & 0.0704225 & 0.1056338 & 2.6 & 0.3846154 \\
\hline 9 & 1.5 & 14.6 & 0.0684932 & $\mathbf{0 . 1 0 2 7 3 9 7}$ & 2.8 & 0.3571429 \\
\hline 10 & 1.8 & 15.6 & 0.0641026 & 0.1153846 & 4 & 0.25 \\
\hline 11 & 1.8 & 16 & 0.0625 & 0.1125 & 4 & 0.25 \\
\hline 12 & 2 & 16 & 0.0625 & 0.125 & 4 & 0.25 \\
\hline 13 & 2.1 & 18 & 0.0555556 & 0.1166667 & 4 & 0.25 \\
\hline 14 & 2.2 & 18.6 & 0.0537634 & 0.1182796 & 4.6 & 0.2173913 \\
\hline 15 & 2.2 & 20 & 0.05 & 0.11 & 4.8 & 0.2083333 \\
\hline 16 & 2.2 & 20.2 & 0.049505 & 0.1089109 & 4.8 & 0.2083333 \\
\hline 17 & 2.3 & 20.4 & 0.0490196 & 0.1127451 & 6 & 0.1666667 \\
\hline 18 & 2.3 & 20.6 & 0.0485437 & 0.1116505 & 6.6 & 0.1515152 \\
\hline 19 & 2.4 & 20.8 & 0.0480769 & 0.1153846 & 6.8 & 0.1470588 \\
\hline 20 & 2.3 & 22 & 0.0454545 & 0.1045455 & 8.6 & 0.1162791 \\
\hline 21 & 2.3 & 24 & 0.0416667 & 0.0958333 & 8.8 & 0.1136364 \\
\hline 22 & 2.3 & 28.6 & 0.034965 & 0.0804196 & 10.2 & 0.0980392 \\
\hline 23 & 2.4 & 30.6 & 0.0326797 & 0.0784314 & 12.4 & 0.0806452 \\
\hline 24 & 2.8 & 34.6 & $\mathbf{0 . 0 2 8 9 0 1 7}$ & 0.0809249 & 14 & 0.0714286 \\
\hline
\end{tabular}

From linear regression equation;

By comparison with equation 1;

$$
y=m x+c \equiv \boldsymbol{y}=0.169 x+0.073
$$

$$
\begin{gathered}
\frac{1}{\mu_{\max }}=c=\text { intercept } ; \text { i.e. } \mu_{\max }=\frac{1}{c}=\frac{1}{0.073}=\mathbf{1 3 . 7 0 \text { day } ^ { - 1 }} \\
\text { and } ; \frac{K_{s}}{\mu_{\max }}=m ; K_{s}=\mu_{\max } * m \\
\therefore K_{s}=13.70 * 0.169=2.32 \mathrm{mg} / \mathbf{L}
\end{gathered}
$$




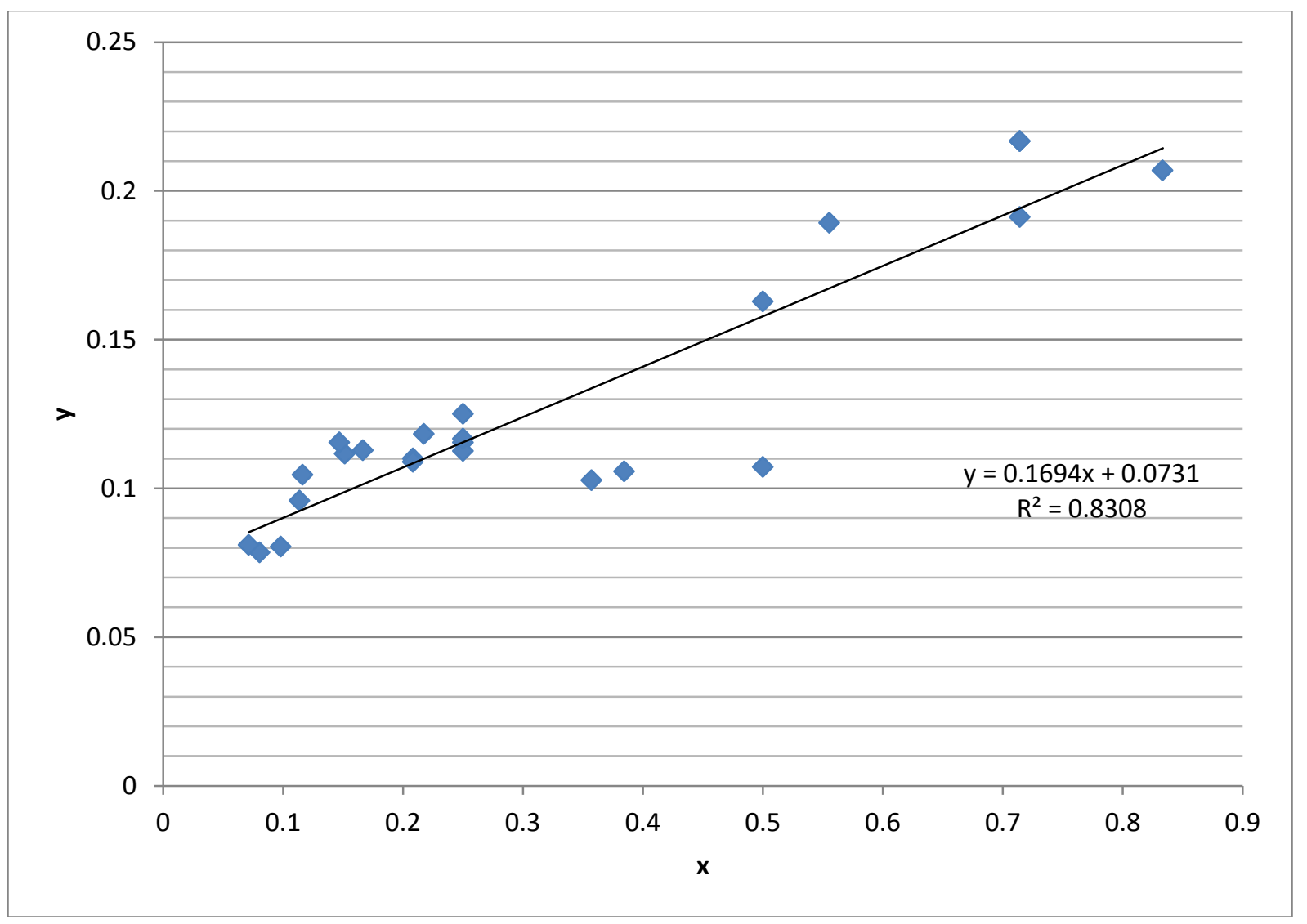

Fig. 4: A Plot of $y=\frac{x V}{Q\left(s_{i}-S_{e}\right)}(/ \mathrm{L} . \mathrm{d} / \mathrm{mg})$ Versus $x=\frac{1}{s_{e}}(\mathrm{~L} / \mathrm{mg})$ for Reactor 3

Substituting $\mu_{\max }$ and $\mathrm{k}_{\mathrm{s}}$ into equation 2 gives;

$$
r_{A}=\frac{d s}{d t}=\frac{Q}{V}\left(S_{i}-S_{e}\right)=\frac{\mu_{\max } * S_{e}}{K_{s}+S_{e}}=\frac{13.70 * S_{e}}{2.32+S_{e}}
$$


3.2 Model Verification

Table 6: Simulation for Reactor 1

\begin{tabular}{|c|c|}
\hline $\begin{array}{c}\text { Observed } y \\
x_{i} \cdot V \\
Q\left(S_{i}-S_{e}\right) \\
(\mathrm{mg} / \mathrm{L.d})\end{array}$ & $\begin{array}{c}\text { Simulated } y^{\prime} \\
\frac{x_{i} \cdot V}{Q\left(S_{i}-S_{e}\right)} \\
(\mathrm{mg} / \mathrm{L.d})\end{array}$ \\
\hline 0.1184211 & 0.11456 \\
\hline 0.1153846 & 0.114208 \\
\hline 0.1666667 & 0.137882 \\
\hline 0.1578947 & 0.135333 \\
\hline 0.1568627 & 0.131 \\
\hline 0.125 & 0.127455 \\
\hline 0.1307692 & 0.1232 \\
\hline $\mathbf{0 . 1 3 3 3 3 3 3}$ & 0.119857 \\
\hline 0.1285714 & 0.125214 \\
\hline 0.1266667 & 0.116375 \\
\hline 0.11875 & 0.114941 \\
\hline 0.1086957 & 0.113667 \\
\hline 0.1075269 & 0.112526 \\
\hline $\mathbf{0 . 1 0 6 3 8 3}$ & 0.10825 \\
\hline 0.105 & 0.105929 \\
\hline 0.1029412 & 0.105448 \\
\hline 0.1019417 & 0.104188 \\
\hline 0.1057692 & 0.103471 \\
\hline 0.1057692 & 0.1024 \\
\hline 0.1017699 & 0.101286 \\
\hline 0.0950413 & 0.100864 \\
\hline 0.0927419 & 0.100478 \\
\hline 0.0923077 & 0.099222 \\
\hline 0.0916031 & 0.09819 \\
\hline
\end{tabular}

Fig. 5: A Scatter plot of Observed y versus Simulated $y^{\prime}$ for Reactor 1

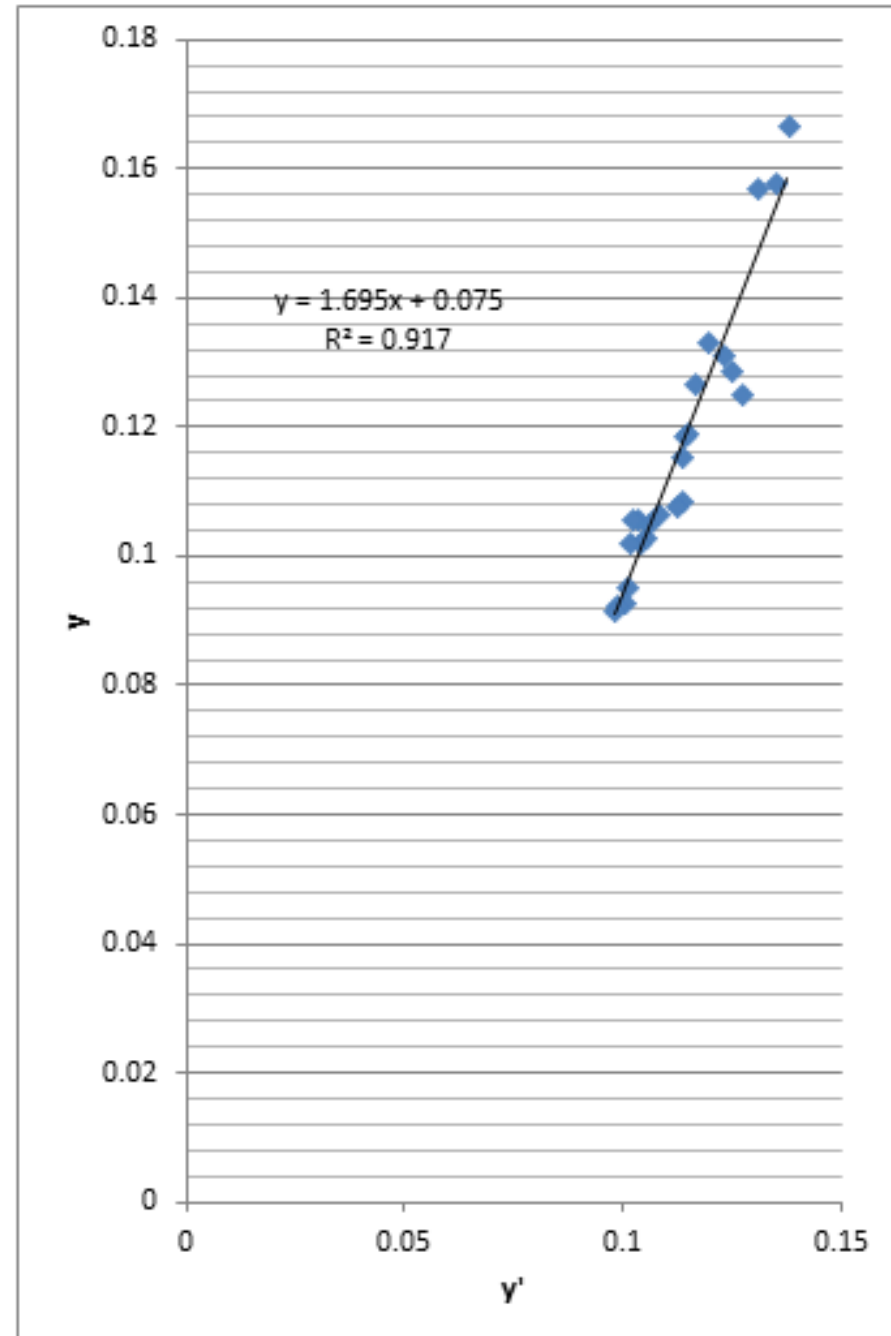

The coefficient of determination, $\mathrm{R}^{2}$ for reactor 1 in Monod Model yielded 0.916, suggesting a satisfactory fitting of the developed model. 
Table 7: Simulation for Reactor 2

\begin{tabular}{|c|c|}
\hline $\begin{array}{c}\text { Observed } y \\
x_{i} \cdot V \\
Q\left(S_{i}-S_{e}\right) \\
(\mathrm{mg} / \mathrm{L.d})\end{array}$ & $\begin{array}{c}\text { Simulated } y^{\prime} \\
\frac{x_{i} \cdot V}{Q\left(S_{i}-S_{e}\right)} \\
(\mathrm{mg} / \mathrm{L.d})\end{array}$ \\
\hline 0.15625 & 0.178 \\
\hline 0.1538462 & 0.178 \\
\hline 0.2205882 & 0.17175 \\
\hline 0.2142857 & 0.17175 \\
\hline 0.1632653 & 0.161333 \\
\hline $\mathbf{0 . 1 3 3 3 3 3 3}$ & 0.146182 \\
\hline 0.1232877 & 0.121367 \\
\hline 0.1216216 & 0.135692 \\
\hline 0.12 & 0.131571 \\
\hline 0.1282051 & 0.128 \\
\hline 0.1176471 & 0.124875 \\
\hline 0.1157895 & 0.123455 \\
\hline 0.1073171 & 0.122118 \\
\hline 0.1223404 & 0.119667 \\
\hline 0.115 & 0.113714 \\
\hline 0.1176471 & 0.10925 \\
\hline 0.1165049 & 0.103862 \\
\hline 0.1153846 & 0.100727 \\
\hline 0.1153846 & 0.097231 \\
\hline 0.1017699 & 0.09675 \\
\hline 0.092 & $\mathbf{0 . 0 9 5 8 5 7}$ \\
\hline 0.0871212 & 0.092151 \\
\hline 0.0872727 & 0.090931 \\
\hline 0.0928571 & 0.087036 \\
\hline
\end{tabular}

Fig. 6: A Scatter plot of Observed y versus Simulated $y^{\prime}$ for Reactor 2

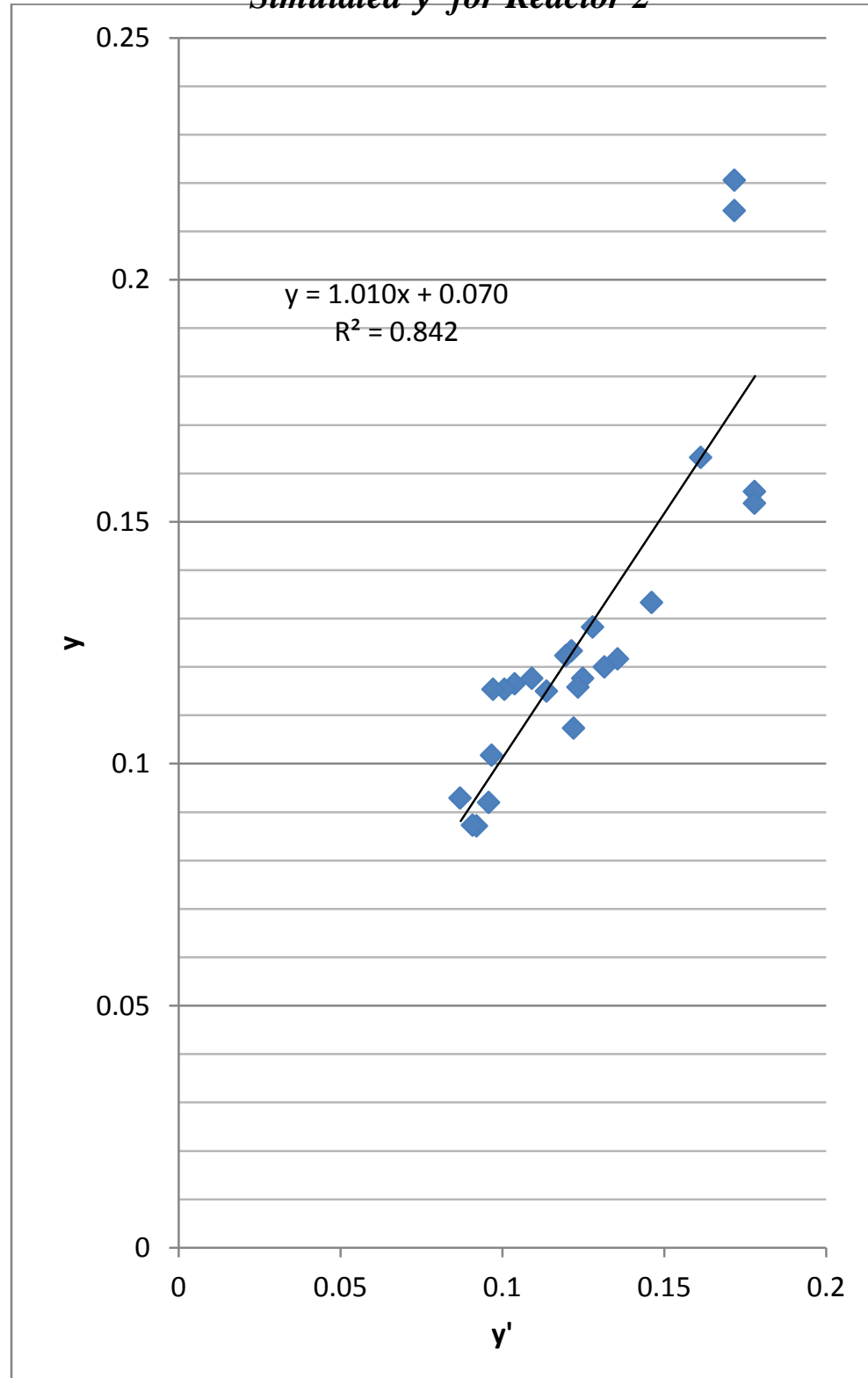

The coefficient of determination, $\mathrm{R}^{2}$ for reactor 2 in Monod Model yielded 0.842 , suggesting a satisfactory fitting of the developed model. 
Table 8 : Simulation for Reactor 3

\begin{tabular}{|c|c|}
\hline $\begin{array}{c}\text { Observed } y \\
x_{i} \cdot V \\
Q\left(S_{i}-S_{e}\right) \\
(\mathrm{mg} / \mathrm{L.d})\end{array}$ & $\begin{array}{c}\text { Simulated } y^{\prime} \\
\frac{x_{i} \cdot V}{Q\left(S_{i}-S_{e}\right)} \\
(\mathrm{mg} / \mathrm{L} . \mathrm{d})\end{array}$ \\
\hline 0.2068966 & 0.213833 \\
\hline 0.2166667 & 0.193714 \\
\hline 0.2166667 & 0.193714 \\
\hline 0.1911765 & 0.193714 \\
\hline 0.1891892 & 0.166889 \\
\hline 0.1627907 & 0.1575 \\
\hline 0.1071429 & 0.1575 \\
\hline 0.1056338 & 0.138 \\
\hline 0.1027397 & $\mathbf{0 . 1 3 3 3 5 7}$ \\
\hline 0.1153846 & 0.11525 \\
\hline 0.1125 & 0.11525 \\
\hline 0.125 & 0.11525 \\
\hline 0.1166667 & 0.11525 \\
\hline 0.1182796 & 0.109739 \\
\hline 0.11 & 0.108208 \\
\hline 0.1089109 & 0.108208 \\
\hline 0.1127451 & 0.101167 \\
\hline 0.1116505 & 0.098606 \\
\hline 0.1153846 & 0.097853 \\
\hline 0.1045455 & 0.092651 \\
\hline $\mathbf{0 . 0 9 5 8 3 3 3}$ & 0.092205 \\
\hline 0.0804196 & 0.089569 \\
\hline 0.0784314 & 0.086629 \\
\hline 0.0809249 & 0.085071 \\
\hline
\end{tabular}

4. Conclusions

Coefficient of determination $\mathrm{R}^{2}$ was used to verify the respective models. The reactors with aspect ratios of $(53: 16: 30 ; 4: 1: 1 ; 10: 3: 6)$ yielded specific growth rate $\boldsymbol{\mu}_{\max }$ and half saturation constant $\boldsymbol{k}_{\boldsymbol{s}}$ for noninhibited cassava wastewater treatment process as follows:

Monod model yielded:

Reactor $1-\left(\boldsymbol{\mu}_{\max }=\mathbf{1 0 . 8 7} \mathbf{d a y}^{-1} ; \quad \boldsymbol{k}_{\boldsymbol{s}}=\right.$ $\left.0.87 \mathrm{mgCODL}^{-1} ; R^{2}=0.917\right)$

Reactor 2-( $\boldsymbol{\mu}_{\max }=\mathbf{1 2 . 8 2} \operatorname{day}^{-1} ; \quad \boldsymbol{k}_{\boldsymbol{s}}=$
Fig. 7: A Scatter plot of Observed y versus

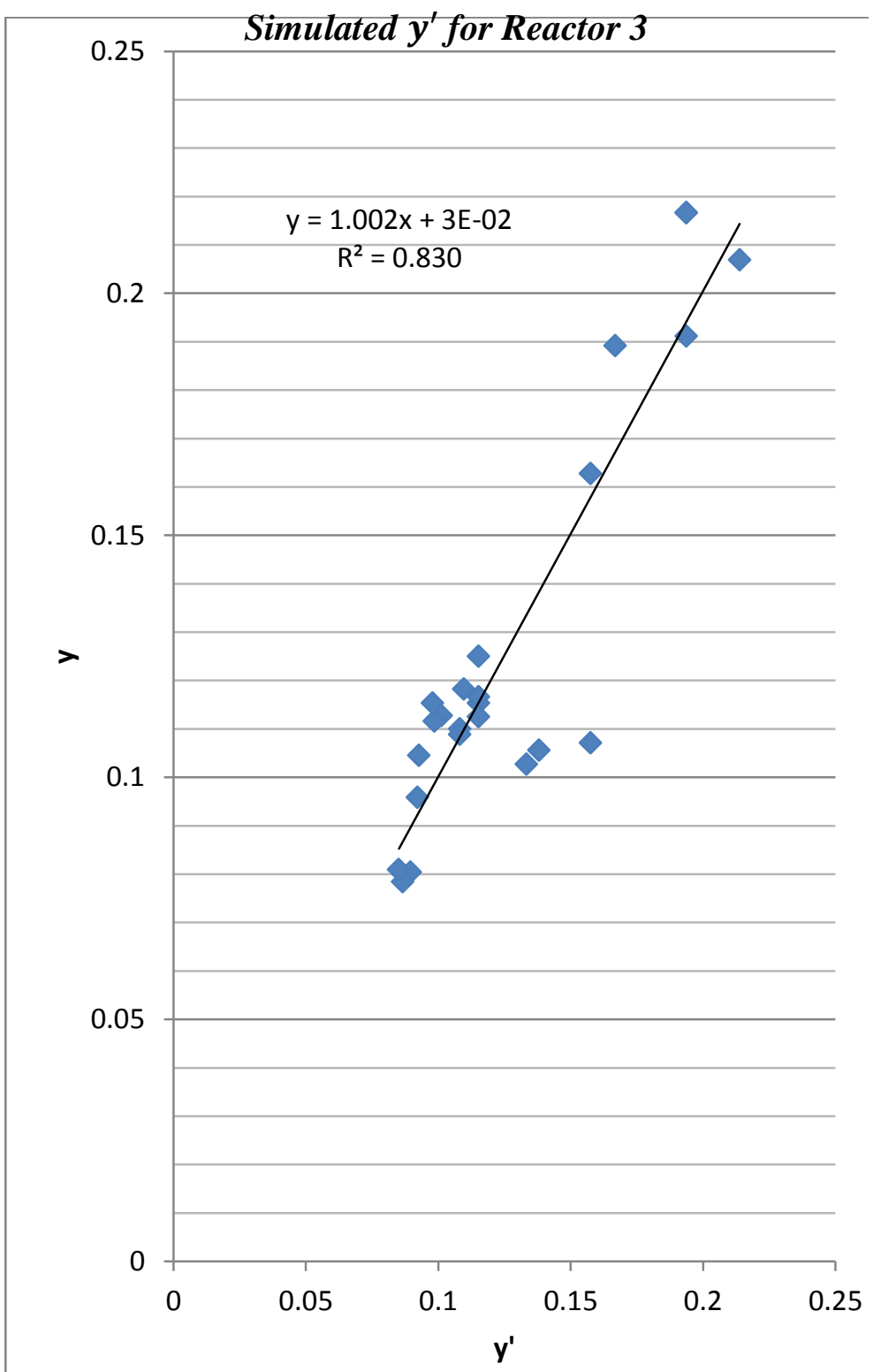

$\left.1.92 \mathrm{mgCODL}^{-1} ; R^{2}=0.842\right)$

Reactor 3- $\left(\boldsymbol{\mu}_{\max }=\mathbf{1 3 . 7 0} \mathrm{day}^{-1} ; \boldsymbol{k}_{\boldsymbol{s}}=\right.$ $\left.2.32 \mathrm{mgCODL} \mathbf{L}^{-1} ; \boldsymbol{R}^{2}=\mathbf{0 . 8 3 0}\right)$. The evaluation showed that the kinetic parameters developed vary within acceptable limits for any size of reactor (physical model) considered, thus paving way for application in large treatment plants.

The coefficient of determination, $\mathrm{R}^{2}$ for reactor 3 in Monod Model yielded 0.830, suggesting a satisfactory fitting of the developed model. 
However, investigations of the hydrodynamics to date have not taken into account other factors, which include biogas mixing effects, viscosity changes due to extra- cellular polymer production, biomass particle size and the rate of solid particles/biomass within the reactor. It is suggested that further studies should thus be carried out.

\section{REFERENCES}

1) Lawrence, J.H. and Moore, L.M. (2005). United States Department of Agriculture Plant Guide. Cassava, Manihot esculenta Crantz. USDA: Washington, DC, 2005. http://plants.usda.gov/plantguide/doc/pg_maes.doc (accessed March 24, 2008)

2) Kawano, K. (2003). Thirty years of cassava breeding for productivity - Biological and social factors for success. Crop Science, 43: 1325-1335.

3) Adeniji, A.A., Ega, L.A., Akoroda, M.O., Ugwu, B.O. and Balogun, A. (2001). Global strategy for cassava development. Nigeria: A country case study.

4) IITA International Inst of Trop Agric (1990). Cassava in tropical Africa-A reference manual. Ibadan, Nigeria: International Institute of Tropical Agriculture.

5) Ugwu, B.O. (1996). Increasing cassava production in Nigeria and prospects for sustaining the trend. Outlook on Agric. 25(3):179-185.

6) Eggleston, G., Onwuka, P.E. and Ihedioha, O.D. (1992). Development and evaluation of products from cassava flour as a new alternative to wheat bread. J. Sci. Food Agric. 59:377-385.
7) Santana, M.A., Vásquez, V., Matehus, J.and Aldao, R.A (2002). Linamarase expression in cassava cultivars with roots of low- and high-cyanide content. Plant Physiol. 129:16861694.

8) Shittu, T.A., Sanni, L.O., Awonorin, S.O., Maziya-Dixon, B. and Dixon, A. (2007).Effect of genotype on the flour making properties of some CMD-resistant varieties of cassava. Food chem. 101;1634-1643.

9) Onitilo, M.O., Sanni, L.O., Oyewole, O.B. and MaziyaDixon, B. (2007). Physico-chemical and functional properties of sour starches from different cassava varieties. Int. J. Food Proper. 10:607-620.

10) Cooke, R.D. and Maduagwu, E.N. (1978). The effects of simple processing on the cyanide content of cassava chips. Journal of Food Technology, 13: 299-306.

11) Nambisan, B. and Sundaresan, S. (1985). Effect of processing on the cyanoglucoside content of cassava. J. Sci. Food Agric. 36:1197-1203.

12) Udensi, E.A., Ukozor, A.U. and Ekwu, F.C. (2005). Effect of fermentation, blanching, and drying temperature on the functional and chemical properties of cassava flour. Int. J. Food Properties 8:171-177.

13) Draaijer, H., Maes, J.A., Schaapman, J.E. and Khan, A. (1992). Performance of 5MLD UASB Reactor For Sewage Treatment at Kanpor, India, Water Sci, Technol. 23(7), 123 133

14) Angenent, L. T., Abel, S. J. and Sung, S. (2002). Effect of an organic shock load on the stability of an anaerobic migrating blanket reactor. J. Environ. Eng., 128 (12): 1109-1120.

15) Hasyimoto, A.G., Roman, L. and Hruska, U.S. (1982) "Methane from cattle waste effects of temperature, hydraulic retention time, and influent substrate concentration on kinetic parameter, Biotech, Bioeng. 26, 2039-2052. 